VOX PATRUM 26 (2006) t. 49

Reiner KACZYNSKI

(München, LMU)

\title{
MYSTAGOGIE: EIN LITURGISCHES BILDUNGSKONZEPT DER ALTEN KIRCHE
}

Anlässlich seiner Amtseinführung hat Papst Benedikt XVI. am 24. April 2005 eine Homilie gehalten, die die zum Gottesdienst Versammelten aufhorchen ließ. Es handelte sich um keine neue Auslegung der bekannten Abschnitte der neutestamentlichen Schriften über den Primat; auch wollte er nicht sein Regierungsprogramm vorstellen, vielmehr ging es ihm um den Versuch, ,einfach die beiden Zeichen - Pallium und Fischerring - auszulegen, mit denen die In-Dienst-Nahme für die Nachfolge des heiligen Petrus liturgisch dargestellt wird; beide Zeichen spiegeln übrigens auch genau das, was in den Lesungen dieses Tages gesagt wird"1.

„Das Pallium ist ein Gewebe aus reiner Wolle, das mir um die Schultern gelegt wird. Dieses uralte Zeichen, das die Bischöfe von Rom seit dem 4. Jahrhundert tragen, mag zunächst einfach ein Bild sein für das Joch Christi, das der Bischof dieser Stadt, der Knecht der Knechte Gottes, auf seine Schultern nimmt. Das Joch Gottes - das ist der Wille Gottes, den wir annehmen"2, sagte der Papst.

Die Übergabe des Fischerringes ist das zweite Zeichen, mit dem die Einsetzung in das Petrusamt dargestellt wird. Dieses zweite Zeichen des Petrusdienstes wird dem Papst vom Dekan des Kardinalskollegiums überreicht. Dazu sagte der Papst: „Auch heute ist es der Kirche und den Nachfolgern der

${ }^{1}$ Ansprache von Benedikt XVI. (24. IV 2005), in: „Verlautbarungen des Apostolischen Stuhls" 2005, n. 168, 32.

${ }^{2}$ Der ranghöchste Kardinaldiakon spricht, wenn er dem Papst das Pallium anlegt: „Der Gott des Friedens, der den großen Hirten der Schafe, unseren Herrn Jesus Christus, von den Toten heraufgeführt hat, er verleihe dir das Pallium, das wir vom Grab des Apostels Petrus genommen haben. Ihm hat der gute Hirte die Sorge für seine Lämmer und Schafe anvertraut. Heute folgst du dem Petrus im Bischofsamt dieser Kirche, die er zusammen mit dem Apostel Paulus begründet hat, nach. Der Geist der Wahrheit, der vom Vater ausgeht, schenke dir in reichem Maß Inspiration und Unterscheidungsgabe für deinen Dienst, die Brüder in der Einheit des Glaubens zu stärken" (Officium de liturgicis celebrationibus Summi Pontificis, Ordo Rituum pro Ministerii Petrini initio Romae Episcopi, Città del Vaticano 2005, n. 51, p. 63). 
Apostel aufgetragen, ins hohe Meer der Geschichte hinauszufahren und die Netze auszuwerfen, um Menschen für das Evangelium [...] zu gewinnen"3.

Mit der Deutung der beiden wichtigsten Zeichen seines petrinischen Dienstes in der Homilie folgte der Papst einer Anregung seines unmittelbaren Vorgängers, Johannes Pauls II. Dieser hatte in seinem Apostolischen Schreiben vom 4. Dezember 2003 „Spiritus et Sponsa” anlässlich des 40. Jahrestages der Veröffentlichung der Liturgiekonstitution des Zweiten Vatikanums angeregt, dass die Bischöfe die in der Zeit der Kirchenväter so bewährte Mystagogie sich aneignen und pflegen sollten. Damit erinnerte Papst Johannes Paul II. an die Liturgiekonstitution des Zweiten Vatikanums, die die Notwendigkeit liturgischer Bildung nicht nur des Klerus ${ }^{4}$, sondern auch der übrigen Gläubigen ${ }^{5}$ betont, und er verweist auf deren in der Pastoral der Alten Kirche praktizierte Form: die Mystagogie ${ }^{6}$. Diese besagt: Hinführung, Geleit, Erziehung $(\dot{\alpha} \gamma \omega \gamma \eta \dot{)})$ der durch Taufe, Firmung und Eucharistie in die Gemeinschaft der Gläubigen bereits Eingegliederten zu (Verständnis und Vollzug) der im Gottesdienst gefeierten Mysterien - Anleitung zu einem von diesem geprägten Leben - durch bereits Eingeweihte („Mysten”), zumeist Kleriker.

\section{DIE CHRISTLICHE MYSTAGOGIE VOM ALTERTUM (3./4. JH.) BIS HEUTE}

Die christliche Mystagogie entwickelte sich in der Alten Kirche im 3./4. Jh. im Zusammenhang mit der Feier der christlichen Initiation, und zwar mit der 3. Phase dieser über Monate hin sich erstreckenden Initiationsfeiern. Ihre wichtigsten Vertreter lebten und hielten ihre „mystagogischen Homilien” bzw. „mystagogischen Katechesen” im 4. Jh.: im Osten der Kirche Asterius der Sophist († nach 341) aus Kappadokien, der in der diokletianischen Verfolgung vom Christentum abgefallen war und sich nach seiner Wiederaufnahme in die

${ }^{3}$ Ibidem, n. 54, p. 65-67. Der Dekan des Kardinalskollegium spricht bei der Überreichung des Fischerringes: „Heiliger Vater, der Bischof und Hirte unserer Seelen, Christus, der Sohn des lebendigen Gottes, der auf einem Felsen seine Kirche erbaut hat, er verleihe dir den Siegelring des Fischers Petrus, der seine Hoffnung auf dem See von Galiläa erfahren hat und dem Jesus, der Herr, die Schlüssel des Himmelreiches anvertraut hat. Dem heiligen Apostel Petrus folgst du heute nach im Bischofsamt dieser Kirche, die den Vorsitz führt in der Einheit der Liebe gemäß der Lehre des heiligen Apostels Paulus; der Geist der Liebe, der in unsere Herzen ausgegossen ist, durchdringe dich mit Kraft und Milde bei deinem Dienst, alle Christgläubigen in der Einheit der Gemeinschaft zu bewahren".

${ }^{4}$ Vgl. Vaticanum II, Constitutio ,Sacrosanctum Concilium” 14.

5 Vgl. ibidem 19.

6 Vgl. Johannes Paul II., Apostolisches Schreiben „Spiritus et Sponsa” vom 4. Dezember 2003 anlässlich des 40. Jahrestages der Veröffentlichung der Liturgiekonstitution „Sacrosanctum Concilium” 12, „Notitiae” 39 (2003) 579. 
Kirche vergeblich um ein Bischofsamt bemühte; sodann Kyrill von Jerusalem $(† 387)$, ferner in der Zeit, in der er als Presbyter der Kirche von Antiochien angehörte, bevor er am 26. Februar 398 Bischof von Konstantinopel wurde, Johannes Chrysostomus († 407) und dessen Freund Theodor von Mopsuestia ( $† 428)$; für den Westen der Kirche sind zu erwähnen Zeno von Verona ( $†$ um 341) und Ambrosius von Mailand († 397).

Nach dem Rückgang der Erwachseneninitiation in der westlichen Kirche ging die gestufte Eingliederung in die Kirche - mit Präkatechumenat - also „Vorkatechumenat" als erster Kontaktaufnahme mit der Kirche, Katechumenat als Zeit der entfernten und Photizominat als Zeit der näheren Vorbereitung auf die Feier der Sakramente bzw. als ,Zeit der Läuterung und Erleuchtung", schließlich mit der Feier der Initiationssakramente, der Sakramente der Eingliederung in die Kirche, und anschließend der „Zeit der Einübung und Vertiefung", eben der Mystagogie, weitgehend verloren. Vor allem konnte von einer Mystagogie im Sinne der in der Alten Kirche vorgesehenen Zeit der Einübung und Vertiefung in die gefeierte Liturgie der Initiationssakramente nicht mehr die Rede sein.

Der nach dem Zweiten Vatikanischen Konzil erarbeitete, am 16. Januar 1972 veröffentlichte und derzeit geltende Ordo initiationis christianae adultorum hat zumindest im deutschen Sprachgebiet noch keine nachhaltige Erneuerung bewirkt. Eine angepasste Fassung von Kapitel I. dieses Ordo ist als Manuskriptausgabe zur Erprobung, von den Liturgischen Instituten Deutschlands, Österreichs und der Schweiz herausgegeben, im Jahr 2001 erschienen und wird da und dort mehr oder weniger zaghaft in die liturgische Praxis umgesetzt: Der Titel dieses Buches lautet: Die Feier der Eingliederung Erwachsener in die Kirche. Grundform (Trier 2001). Die Diktion in diesem Buch ist leider gelegentlich unbefriedigend und missverständlich. Das Vorwort schon gibt als Inhalt des Buches an „Die Grundordnung des Erwachsenenkatechumenats mit seinen liturgischen Feiern in einer neuen überarbeiteten Fassung". Indem man nur vom „Katechumenat” spricht, macht man deutlich, dass man wie in anderen Ländern auch, die ausschließlich den Katechumenat betonen, dieses für das wichtigste Element des Initiationsvorgangs hält.

Balthasar Fischer, der im Jahr 2001 verstorbene Trierer Liturgiewissenschaftler, der anlässlich eines Studienaufenthalts in der USA dort die Einführung der neuen Ordnung für die Erwachsenen-Initiation erlebt hatte, berichtete nach seiner Rückkehr in Vorlesungen und Vorträgen immer wieder nicht nur von den guten Erfolgen mit dem Katechumenat, sondern auch von den Schwachpunkten im Umgang mit der neuen Ordnung ${ }^{7}$. Er hat mehrfach erfah-

${ }^{7}$ Vgl. B. Fischer, Das amerikanische Beispiel. Die Rezeption des Ritus der Erwachseneninitiation von 1972 in den Vereinigten Staaten, LJ 37 (1987) 67-74; idem, Zur endgültigen Gestalt des Ritus der Erwachseneninitiation in den USA ab 1.9.1988, LJ 38 (1988) 223-230; vgl. auch idem, Freige- 
ren müssen, dass die Ordnung für den Katechumenat mit großer Begeisterung in den Gemeinden aufgenommen wurde, die Kandidaten auf den Empfang der Sakramente bestens vorbereitet wurden, dass man jedoch nach dem Osterfest die neu in die Kirche aufgenommenen Christen weitgehend sich selbst überließ. Ein selbst erlebtes Beispiel schildert der Verfasser im ersten seiner erwähnten Berichte folgendermaßen:

„Am tiefsten bewegt hat mich die Geschichte vom Detroiter Hüttenarbeiter Terry, die mir Schwester Arlene, Leiterin des Liturgiedepartements im Erzbischöflichen Ordinariat Detroit noch in der Wartezeit auf dem Flugplatz erzählte. Terry war eines Tages in einer der Detroiter Pfarreien Katechumene geworden und begab sich mit allem heiligen Eifer auf den Weg, der in den Mysterien der Eingliederung in der Osternacht mündet. Aber für das, was nun folgen sollte, hatte Terry kein Glück. Der Pfarrer seiner Pfarrei war - wie manche seiner Kollegen - unsicher, was er mit den doch an ihr Ziel gekommenen Kandidaten nach Ostern noch anfangen solle, und aus dieser Unsicherheit ließ er die ganze am Ende des Eingliederungsprozesses vorgesehene Phase der Mystagogie fallen. Terry fühlte, dass ihm hier etwas wesentliches fehlte. Bei einer späteren Fragebogenaktion hat er das, was er damals empfand etwa so ausgedrückt: Er sei sich vorgekommen, wie ein Mann, den man auf einen hohen Gipfel geführt und zu dem man dann gesagt habe: Alles, was du da unten siehst, ist dein. Aber es sei niemand da gewesen, der ihm gezeigt habe, wie er sich in dieser seiner neuen Welt bewegen solle. Über diesen Ausfall sei er enttäuscht gewesen, ja er habe manchmal gedacht, ob der ganze Weg zum Gipfel mit all seinem Enthusiasmus nicht eine Enttäuschung gewesen sei. Terry hat in dieser Aufzeichnung nachbeschrieben, was er dann getan hat. Er hat in seiner Verzweiflung seinen Taufpaten (bezeichnenderweise nicht den Taufpriester) angerufen, auch er von Beruf Hüttenarbeiter. Dieser hat auf den Anruf seines «Patenkindes» alles stehen- und fallen lassen, was er gerade zu tun hatte, hat sich in sein Auto gesetzt und ist gekommen. Den stundenlangen Gesprächen, die dann folgten, schreibt Terry, habe er nach Gottes Gnade zu verdanken, dass er seines Christseins wieder froh geworden sei" 8 .

Daraus wird deutlich, dass nicht erst von Seiten des Klerus, sondern schon von Seiten der in die Kirche Aufgenommenen die Notwendigkeit einer geistlichen Erschließung des liturgischen Geschehens erkannt wurde. Wie das geschah, lässt sich anschaulich machen mit einem Blick auf die Verkündigungspraxis des Johannes Chrysostomus. Im Jahrzehnt zwischen 387 und 397 hielt Johannes mehrere mystagogische Katechesen ${ }^{9}$.

legter Zugang. Das Aufleben des Katechumenats in den USA - ein Bericht, „Gottesdienst” 20 (1986) 33-36, bes. 35 .

${ }^{8}$ Vgl. Fischer, Das amerikanische Beispiel, p. 73.

9 Die erste Katechese wurde am Mittwoch in der Karwoche des Jahres 387 gehalten und wird im Allgemeinen als 21. Säulenhomilie gezählt, vgl. Joannes Chrysostomus, Catecheses baptismales. 


\section{JOHANNES CHRYSOSTOMUS ALS MYSTAGOGE}

Zu Beginn des Jahres 386 war Johannes von Bischof Flavian im Alter von etwa 37 Jahren zum Presbyter der Antiochenischen Kirche geweiht worden. Erst von dieser Zeit an hatte er das Recht, Homilien und Katechesen zu halten. Seine erste Predigt, die in der Literatur gelegentlich als seine „Primizpredigt" bezeichnet wird, hielt er am Tag seiner Priesterweihe unmittelbar nachdem diese vollzogen worden war $^{10}$. Diese Predigt kann als programmatisch für seinen ganzen künftigen Dienst gelten. Denn darin versteht er die Verkündigung des Wortes Gottes nicht nur als Dienst an den Menschen und für die Menschen, sondern auch als kultischen Dienst vor und für Gott, als Opfer ${ }^{11}$. Diese Auffassung von seinem Predigtdienst behielt er sein ganzes weiteres Leben bei. Auch als Bischof verstand er die Verkündigung des Wortes Gottes als Mitte der zur Ehre Gottes gefeierten Liturgie.

Nach seiner Ordination zum Presbyter konnte Johannes seine rhetorische Begabung und seine umfassende Schriftkenntnis in den Dienst der Vermittlung des Wortes Gottes stellen, den er zunächst 12 Jahre lang in der Kirche von Antiochien versah. Außer seiner Predigttätigkeit sind keine weiteren besonderen seelsorglichen Initiativen des Kirchenvaters überliefert. Die Predigten und Katechesen machten ihn weit über seine Antiochenische Gemeinde hinaus bekannt und berühmt. Sie wurden zum großen Teil mitstenographiert, von ihm selbst überarbeitet und schließlich veröffentlicht bzw. zur Veröffentlichung freigegeben. Diese Predigten sind der Grund, weshalb man ihm seit dem 5.l 6. Jh. den Beinamen Chrysostomus („Goldmund”) gab, der schließlich, was ähnlich bei nur wenigen anderen Kirchenvätern der Fall war, zu seinem Eigennamen wurde.

Taufkatechesen, übersetzt und eingeleitet von R. Kaczynski, Fontes Christiani (= FCh) 6/1-2, Freiburg - Basel -Wien 1992. Cat. 1, p. 30-34 und 50-51 (Einleitung), 110-149 (Text). Die cat. $2 /$ 1-3 wurden während der Quadragesima 388 an die Taufbewerber gerichtet. Cat. 2/4 wurde in der Osternacht des gleichen Jahres gehalten und ist an die Neugetauften und die ganze übrige Gemeinde gerichtet. Cat. 3/1-7 hielt Johannes Chrysostomus in einem nicht näher bestimmbaren Jahr zwischen 389 und 397, cat. 3/1 und 3/2 am Beginn und Ende einer Quadragesima; cat. 3/3 wurde in der Osternacht, cat. 3/4-7 wurden während der Osterwoche eben dieses Jahres gehalten. Nur die Hälfte der erwähnten Katechesen, nämlich cat. 2/4 bis 3/3-7 können demnach als mystagogische Katechesen im strengen Sinn bezeichnet werden. Die Katechesen 3/1-7 wurde erst 1955 durch

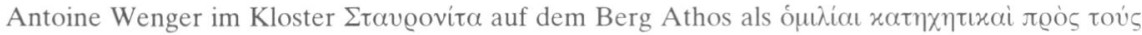

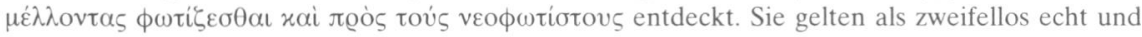
wurden in der Reihe Sources Chrétiennes von A. Wenger erstmals zusammenhängend veröffentlicht (SCh 50: Jean Chrysostome, Huit catéchèses baptismales inédites, Paris 1957). Sie finden sich in Fontes Christiani 6/2, 292-467.

${ }^{10} \mathrm{Vgl}$. Sermo, cum presbyter fuit ordinatus, PG 48, 694-700.

11 Vgl. R. Kaczynski, Das Wort Gottes in Liturgie und Alltag der Gemeinden des Johannes Chrysostomus (FThSt 94), Freiburg - Basel - Wien 1974, 18-21. 
Johannes Chrysostomus hat seine Taufkatechesen in Antiochien nicht als Bischof gehalten, sondern als Presbyter, vermutlich ungefähr zur gleichen Zeit wie sein Studienfreund Theodor, der spätere Bischof von Mopsuestia. Nur die sechs Katechesen, die im strengen Sinn mystagogisch genannt werden können, sollen im Folgenden auf ihren mystagogischen Gehalt hin untersucht werden. Das ist aus der zweiten im Jahr 388 gehaltenen Reihe von vier Katechesen die letzte, also die 4., die Johannes in der Osternacht gehalten hat.

\section{WICHTIGE INHALTE DER „MYSTAGOGISCHEN KATECHESEN” DES JOHANNES CHRYSOSTOMUS}

1. Katechese 2/4. Die erste im strengen Sinn „mystagogische Katechese” von Johannes Chrysostomus ist cat. 2/4, die er in der Osternacht des Jahres 388 gehalten hat. Er spricht allgemein von den Wirkungen der Taufe. Die Tauffeier ist beendet, die Neugetauften sind mit der Gemeinde der schon länger Getauften versammelt, um die österliche Eucharistie zu halten. Chrysostomus ist erfüllt von Freude und Begeisterung. Er preist Gott dafür, dass die Taufe die Menschen zum Leuchten gebracht hat wie Sterne, die heller sind als die am Firmament und darum auch am Tag gesehen werden und als Morgensterne gelten können. Außer dem Sündennachlass erwähnt Johannes zehn weitere Wirkungen der Taufe: Sie macht frei, heilig und gerecht; die Neugetauften sind Söhne, Erben, Brüder Christi, seine Miterben, Glieder seines Leibes, Tempel des Heiligen Geistes und seine Werkzeuge. In diesen zehn Wirkungen sieht er eine Begründung für die Kindertaufe:

„Deswegen taufen wir ja auch kleine Kinder, obgleich sie keine Sünde haben, damit sie geheiligt und gerecht gemacht werden, damit sie die Sohnschaft und das Erbe erhalten, damit sie Brüder und Glieder Christi werden und der Heilige Geist in ihnen Wohnung nimmt"

Und sofort folgt die Anwendung auf das Leben: nun komme es darauf an, dem großen Geschenk entsprechend zu leben.

Mit dem Tauftag ist die Übungszeit vorbei, der Wettkampf, der Kampf gegen den Teufel hat begonnen. Dabei ist Christus nicht unparteiisch wie ein Schiedsrichter, sondern er steht auf der Seite der Getauften. Wenn der Teufel in diesem Kampf siegt, wird er wie einst im Paradies bestraft, setzt sich aber der Christ dank der von Christus erhaltenen Waffen durch, so erhält er den Siegeskranz ${ }^{13}$.

Über die Ausrüstung mit Waffen zum Kampf gegen den Satan hinaus ist dem Christen das eucharistische Mahl bereitet, durch das er sich zum Kampf

12 Vgl. Catechesis 2-4, 6, FCh 6-1, 258-261.

13 Vgl. ibidem 2-4, 8-11, FC 6/1, 260-269. 
stärken und durch das gekräftigt er den Satan überwinden kann ${ }^{14}$. Chrysostomus stellt nun den alttestamentlichen Typos der neutestamentlichen Erfüllung gegenüber. Vom alttestamentlichen Typos her, dem Blut des fehlerlosen Lammes, das, vor dem Auszug aus Ägypten auf die Türpfosten gestrichen, das jüdische Volk rettete, kann man auf die rettende Kraft des Blutes Christi schließen: Denn zusammen mit Wasser kam es aus der Seite des am Kreuz hingerichteten Christus hervor und weist auf die Eucharistie wie das Wasser auf die Taufe hin ${ }^{15}$. Die durch diese beiden Sakramente sich bildende und stets erneuernde Kirche erschuf Christus also aus seiner Seite wie einst Eva aus der Seite Adams erschaffen wurde. Das Blut des vernunftlosen Tieres konnte die vernunftbegabten Menschen retten, nicht weil es Blut war, sondern weil es auf das Blut Christi verwies, dessen Vorausbild es war. Und auch hier wieder die „moralische Anwendung”: Die Größe dieses Geschenkes und der mit Christus geschlossene Vertrag verpflichten zu einem entsprechenden Leben, in dem man sich nicht wieder dem alten Vertrag unterwirft; denn der alte Schuldschein ist durch den Tod Jesu am Kreuz zerrissen ${ }^{16}$.

Gegen Ende dieser Katechese stellt Chrysostomus den Auszug aus Ägypten als Typos dem Geschehen der Taufe gegenüber und weist darauf hin, dass dieses sein Vorausbild, den Typos, in vielfacher Hinsicht übertrifft ${ }^{17}$, wie auch die Eucharistie die atl. Typoi des Manna und des Wassers aus dem Felsen bei weitem überragt. Der Bischof bzw. Christus selbst reicht in der Eucharistiefeier die ewige Speise und lässt aus dem Altar Quellen des Geistes hervorsprudeln. Ein aufrichtiges Herz und ein reines Gewissen sind beim Hinzutreten zum eucharistischen Mahl erforderlich ${ }^{18}$.

2. Katechese 3/3. Die zweite im strengen Sinn mystagogische Katechese hielt Johannes Chrysostomus in der Osternacht eines Jahres zwischen 389 und 397. Der Kirchenvater beginnt wiederum damit, dass er seiner Begeisterung und Freude über die Neugetauften Ausdruck gibt: die Kirche, ihre geistliche Mutter freut sich über ihre Geburt: wenn man sich im Himmel über einen einzigen Sünder, der umkehrt, freut, so muss auf Erden doch die ganze Gemeinde über eine so große Anzahl Neugetaufter in Jubel ausbrechen ${ }^{19}$. Die Gemeinde fordert der Prediger auf, sich den Neugetauften liebevoll zuzuwen$\operatorname{den}^{20}$. Diese sollen sich an Paulus als Vorbild ausrichten und ihn nachahmen. Dann erklärt Chrysostomus der Gemeinde das Geschehen der Taufe ausge-

\footnotetext{
${ }^{14} \mathrm{Vgl}$. ibidem 2/4, 12, FCh 6/1, 268-269.

$15 \mathrm{Vgl}$. ibidem 2/4, 13-23, FCh 6/1, 268-283.

16 Vgl. ibidem 2/4, 20-22, FCh 6/1, 278-282.

17 Vgl. ibidem 2/4, 24-25, FCh 6/1, 282-287.

18 Vgl. ibidem 2/4, 24-26, FCh 6/1, 286-289.

19 Vgl. ibidem 3/3, 1-3, FCh 6/2, 358-361.

${ }^{20} \mathrm{Vgl}$. ibidem 3/3, 4-6, FCh 6/2, 360-363.
} 
hend von der Lesung der Osternachtfeier als Neuschöpfung, auf die die Neugetauften mit einem veränderten Lebenswandel antworten sollen ${ }^{21}$. Mit den entsprechenden Ermahnungen, an dem in der Taufe mit dem Herrn geschlossenen Vertrag festzuhalten, endet diese österliche Homilie ${ }^{22}$.

3. Katechese 3/4. Diese Katechese wurde gehalten in der Osterwoche eines Jahres zwischen 389 und 397.

Chrysostomus geht von dem Gedanken aus, dass die Fastenzeit beendet ist und die Gemeinde nun das „,wahre Fasten” pflegen soll: unmäßiges Essen und Trinken soll sie vermeiden; die „Trunkenheit” im übertragenen Sinn soll die Gemeinde ablegen: Zorn, eitle Ruhmsucht, Überheblichkeit und andere Leidenschaften. Sehr anschaulich schildert der Kirchenvater die Auswirkungen der Trunkenheit und des abstoßenden Anblicks der Betrunkenen, die in einem elenderen Zustand sind als Besessene, denen das Reich Gottes verschlossen bleibt ${ }^{23}$. Der Prediger weiß zwar, dass die Anwesenden von diesen Lastern frei sind; sonst wären sie gar nicht im Gottesdienst ${ }^{24}$. Er kennt aber die menschliche Schwachheit nur zu gut, als dass er nicht wüsste, dass die während der Quadragesima eingehaltene Zucht zu Ostern leicht in Nachlässigkeit umschlägt, enthaltsame Lebensweise in Unmäßigkeit. Abschreckendes Beispiel sind die Israeliten nach ihrer Befreiung aus Ägypten ${ }^{25}$. Wieder wird Paulus als Vorbild erwähnt, bei dem die Taufe eine radikale Veränderung seiner Gesinnung und seines Verhalten bewirkt hat ${ }^{26}$. Wachsamkeit ist von allen gefordert. Die Getauften - Chrysostomus schließt sich selbst ein - mögen ihre Lebensweise ändern ${ }^{27}$.

4. Katechese 3/5. Auch diese Katechese wurde während der Osterwoche eines Jahres zwischen 389 und 397 gehalten. Eine große Menge der Gemeinde hatte sich ins Hypodrom zum Pferderennen begeben, das von unsittlichen Theatervorführungen unterbrochen wurde, anstatt in den Gottesdienst ${ }^{28}$. Die Buße und die Osterfeier sind vergessen. Nach all den Ermahnungen und Aufforderungen zu sittlich einwandfreiem Leben werden sie nun eine größere Strafe zu erwarten haben. Wieder mahnt Chrysostomus zu einer sittlich einwandfreien Lebensführung: Dankbarkeit gegenüber Gott, beim Essen und Trinken nicht über weltliche Dinge zu sprechen, Unmäßigkeit zu meiden, ganz

\footnotetext{
${ }^{21} \mathrm{Vgl}$. ibidem 3/3, 12-16, FCh 6/2, 368-371.

$22 \mathrm{Vgl}$. ibidem 3/3, 17-33, FCh 6/2, 370-385.

23 Vgl. ibidem 3/4, 1-11, FCh 6/2, 386-397.

${ }^{24} \mathrm{Vgl}$. ibidem 3/4, 12-14, FCh 6/2, 396-399.

${ }_{25} \mathrm{Vgl}$. ibidem 3/4, 15-18, FCh 6/2, 398-402.

26 Vgl. ibidem 3/4, 19-23, FCh 6/2, 402-407.

27 Vgl. ibidem 3/4, 24-28, FCh 6/2, 406-411.

${ }^{28}$ Vgl. ibidem 3/5, 1-7, FCh 6/2, 412-419.
} 
allgemein: sich aller bösen Werke zu enthalten, sind seine Forderungen. Man suche vielmehr den Umgang mit Notleidenden oder Gefangenen, wende sich Waisen und Witwen zu, verschwende seine Zeit nicht mit unnützen Zusammenkünften. Wenn man um sein Heil besorgt ist, so ist Vorsicht geboten. Das Heil der leichtsinnigen Mitchristen solle jedem am Herzen liegen. Während der ganzen Osterwoche sollen sie zum Gottesdienst kommen, der wie bei einer Hochzeitsfeier sieben Tage hindurch fortgesetzt wird.

5. Katechese 3/6. Diese Katechese ist ebenfalls während der Osterwoche, vermutlich am Freitag, gehalten worden. Sie beginnt mit Ausführungen über die Märtyrerverehrung. Märtyrer sind geistliche Gestalten, zu deren Gräbern man bei körperlichem und seelischem Leid ohne große Mühen Zuflucht nehmen kann. Sie können durch ihre Fürbitte helfen. Chrysostomus kommt nun auf die für alle in der Welt lebenden Christen geltende Forderung des Apostels zu sprechen, den Sinn auf das Himmlische zu richten (vgl. Kol 3,1 f.) ${ }^{29}$. Die Märtyrer sind darin Vorbilder ${ }^{30}$. Der Brauch, nach der Taufe ein weißes Gewand zu tragen, muss auch in der Gemeinde von Antiochien bekannt gewesen sein. Die Schönheit dieses Gewandes kann man sich durch Gebet, Danksagung für alles Geschenkte und Bitte um dessen Bewahrung erhalten. Zum Gebet muss das Almosen hinzukommen. Als Vorbild nennt Chrysostomus den Hauptmann Cornelius, der trotz seines Militärdienstes ein Leben führte, das von Gebet und reichlichem Almosengeben, geprägt war ${ }^{31}$.

6. Katechese 3/7. Auch diese Katechese wurde während der Osterwoche, vermutlich am Samstag, gehalten. Die Antiochenische Gemeinde hat Gäste in ihrem Gottesdienst: Mönche aus dem Umland Antiochiens. Obwohl sie nur syrisch verstehen und sprechen, begrüßt sie Chrysostomus in griechischer Sprache mit großer Herzlichkeit und lobt sie ob ihrer christlichen Lebensweise. Wie Paulus verdienen sie mit ihrer Hände Arbeit ihren Lebensunterhalt und sammeln Schüler um sich, denen sie die Heilige Schrift vorlesen und erklären. Alle Unmäßigkeit liegt ihnen fern. Leibliche Bedürfnisse stellen sie zurück, irdischen Prunk lehnen sie ab. Sie wissen in ihren irdischen Aufgaben Bescheid, sind aber mit ihren Gedanken ganz bei den überirdischen Dingen ${ }^{32}$. Abraham war für solches Verhalten beispielhaft ${ }^{33}$. Chrysostomus prangert nun das dem Verhalten Abrahams und der anderen Gerechten entgegen gesetzte Verhalten dieser Christen an. Während den Gerechten irdische Güter verheiBen waren und sie nach der himmlischen Wirklichkeit verlangten, haben sie die

\footnotetext{
29 Vgl. ibidem 3/6, 1-16, FCh 6/2, 434-447.

$30 \mathrm{Vgl}$. ibidem $3 / 6,17-19$, FCh 6/2, 446-449.

${ }^{31} \mathrm{Vgl}$. ibidem 3/6, 24-33, FCh 6/2, 452-463.

32 Vgl. ibidem 3/7, 1-6, FCh 6/2, 464-471.

33 Vgl. ibidem 3/7, 7-10, FCh 6/2, 470-475.
} 
Verheißung überirdischer Güter und sind auf irdische ausgerichtet. Flüchtig sind die irdischen Güter, zuverlässig und fest dagegen die Verheißungen Gottes $^{34}$. Darum sollen sich die Neugetauften und die ganze Gemeinde um die bleibenden und unveränderlichen Güter bemühen ${ }^{35}$, täglich am Morgen zu den „Laudes" und am Abend zur „Vesper” in die Kirche kommen, Lobpreis Gottes und Bitte um Gottes Hilfe für den Tag sind die Motive für diese Gebetsgottesdienste $^{36}$.

Abschließend legt Chrysostomus als letzte Ermahnung an die Neugetauften seinen Hörern nochmals die Sorge um die geistlichen Dinge nahe, weil Gott dann auch die materiellen reichlich schenken wird ${ }^{37}$. Die Sorge um das Größte in uns, die Seele, hat Gott uns überlassen, die Sorge für die Bedürfnisse des Leibes übernimmt $\mathrm{er}^{38}$. Vor allem die Neugetauften sollen täglich darauf achten, sich den Glanz ihres geistlichen Gewandes zu bewahren ${ }^{39}$.

\section{DER MYSTAGOGISCHE CHARAKTER DER KATECHESEN DES JOHANNES CHRYSOSTOMUS}

Mit der Darstellung wesentlicher Inhalte ausgewählter Katechesen des Johannes Chrysostomus sollte anschaulich werden, wie ein Priester der Alten Kirche seine Predigttätigkeit verstand und ausübte: nicht nur, aber auch wesentlich als Mystagogie.

Mystagogisch im strengen Sinn sind diese Katechesen insofern, als sie an die durch Taufe, (Firmung) und Eucharistie in der Osternacht neu der christlichen Gemeinde Eingegliederten gerichtet sind und somit vornehmlich in der Osterwoche gehalten wurden. Mystagogisch sind sie dem Inhalt nach, wenn die Erwachsenen über das liturgische Geschehen, genauerhin die Sakramente, die sie empfangen haben unterwiesen werden. Mystagogisch sind sie hinsichtlich der geistlichen Bedeutung, indem hingeführt wird an die Geheimnisse des Glaubens. Somit ist Ziel mystagogischer Katechese die Befähigung zur Feier des Gottesdienstes, besonders der Sakramente in einem reifen Glauben.

Die Betrachtung der Katechesen des Chrysostomus lässt zunächst erkennen, dass sie mystagogischen Charakter haben, auch wenn sie teilweise an die Katechumenen zur Vorbereitung auf die Feier der Eingliederung in die Kirche gerichtet sind. Jedoch ist noch etwas anderes im Blick auf die Katechesen bemerkenswert: es handelt sich stets nicht allein um Mystagogie im strengen

\footnotetext{
${ }^{34}$ Vgl. ibidem 3/7, 14-15, FCh 6/2, 476-478.

Vgl. ibidem 3/7, 16, FCh 6/2, 479-481.

36 Vgl. ibidem 3/7, 17-18, FCh 6/2, 478-481.

37 Vgl. ibidem 3/7, 19-21, FCh 6/2, 482-485.

${ }_{38} \mathrm{Vgl}$. ibidem 3/7, 22-23, FCh 6/2, 484-485.

${ }^{39} \mathrm{Vgl}$. ibidem 3/7, 25, FCh 6/2, 486-487.
} 
Sinn. Chrysostomus kann nicht umhin, den Erklärungen und Deutungen des liturgischen Geschehens Hinweise hinzuzufügen, die den Hörern nahelegen, dass ihnen mit der Feier des Gottesdienstes Aufgaben für eine christliche Lebensführung gestellt sind. Somit kann für seine Predigttätigkeit als charakteristisch gelten, dass er immer Hilfe zum Glauben und zugleich Hilfe zu einem Leben aus dem Glauben geben will. Damit erweist er sich als überzeugter und überzeugender Seelsorger in Verantwortung für die ihm anvertrauten christlichen Gemeinden.

\section{LITURGISCHE BILDUNG HEUTE UNTER BERÜCKSICHTIGUNG FRÜHCHRISTLICHER KATECHETISCHER PRAXIS DES JOHANNES CHRYSOSTOMUS}

Liturgische Bildung heute unter Berücksichtigung frühchristlicher katechetischer Praxis des Johannes Chrysostomus sollte die Zusammengehörigkeit von Mystagogie im strengen Sinn mit der Unterweisung über christliche Lebensführung als dringlich ansehen. Entsprechend der Aussagen des Zweiten Vatikanums ist zuerst für eine gründliche liturgische Ausbildung des Klerus zu sorgen, um ihn so zu befähigen zur Erfüllung seiner unverzichtbaren seelsorglichen Aufgaben: nämlich sich zu bemühen um liturgische Bildung (institutio) mit dem Ziel voller, bewusster und tätiger Teilnahme der Gläubigen an den liturgischen Feiern ${ }^{40}$. Dieses Bemühen findet zunächst seinen Ausdruck in der Katechese bzw. Homilie des Priesters ${ }^{41}$. Es kann jedoch letztlich nur wirksam werden, wenn der Priester selbst dem Gottesdienst in der Weise vorsteht, wie es die letzte Mahnung des Bischofs bei seiner Priesterweihe besagt:

„Bedenke, was du tust,

ahme nach, was du vollziehst,

und stelle dein Leben unter das Geheimnis des Kreuzes"42.

${ }^{40} \mathrm{Vgl}$. Constitutio „Sacrosanctum Concilium” 14-19.

${ }^{41} \mathrm{Vgl}$. hierzu die umfassende Umschreibung der Homilie in der Allgemeinen Einführung in das Römische Messbuch, Nr. 41: „Die Homilie ist ein Teil der Liturgie und wird nachdrücklich empfohlen, denn sie ist notwendig, um das christliche Leben zu stärken. Sie soll unter Berücksichtigung des Mysteriums, das gefeiert wird, und der besonderen Bedürfnisse der Hörer die Schriftlesungen oder andere Texte der Tagesmesse (Ordinarium oder Proprium) unter einem bestimmten Gesichtspunkt auslegen"; vgl. auch R. Kaczynski, Predigten zum Hochgebet, gehalten 1992 (Lesejahr C) in Eglharting (Pfarrei Kirchseeon); idem, ,. Lasst uns singen dem Herrn!” Geistliche Vorträge zu gottesdienstlichen Liedern, München 1997.

42 Pontifikale für die katholischen Bistümer des deutschen Sprachgebietes, Bd 1: Die Weihe des Bischofs, der Presbyter und der Diakone, 2: Die Weihe der Priester, Trier 1994, 94; vgl. ferner R. Kaczynski, ,Seid ihr bereit...?” Geistliche Vorträge zu Diakonen- und Priesterweihe, München 1994, 195-201. 
Wenn der Priester dieser Mahnung folgt, gibt er selbst Zeugnis und ist erst wirklich im Stande, die ihm zur Seelsorge anvertrauten Gläubigen zu überzeugen von der Bedeutung des Gottesdienstes selbst und der Bedeutung, die die gottesdienstliche Feier für das Leben eines Christen hat. Dabei sollte sich der Seelsorger immer dessen bewusst sein, dass sein Bemühen um liturgische Bildung anderer Gläubiger nicht besagt: Formung (formatio) des Einzelnen zum Christen durch ihn, den Seelsorger. Denn Aufgabe des Seelsorgers kann es nur sein, andere anzuregen und durch Unterweisung zu befähigen, instand zu setzen, dass jeder Einzelne eine bewusste und tätige Teilnahme am Gottesdienst und eine dementsprechende Lebensführung als seine eigene Aufgabe übernimmt und in deren Erfüllung sich zu einem mündigen Christen bildet. Auch diese Überlegung stimmt mit dem überein, wie Johannes Chrysostomus Möglichkeiten und Grenzen seines pastoralen Wirkens verstanden hat. Ganz so abwegig erscheint es also wohl nicht, wenn man heute wieder nach der Bedeutung der frühchristlichen Mystagogie als einer Form liturgischer Bildung fragt. Denn auch für die Seelsorgspraxis kann heute gelten:

„Denk an die Tage der Vergangenheit, lerne aus den Jahren der Geschichte!

Frag deinen Vater, er wird es dir erzählen, frag die Alten. Sie werden es dir sagen" ${ }^{, 4}$.

43 Dtn 32, 7. Stundenbuch für die katholischen Bistümer des deutschen Sprachgebietes: Canticum in den Laudes am Samstag der 2. Woche. 